Journal of Patient-Centered

$1-17-2022$

\title{
In Gratitude of 2021 JPCRR Peer Reviewers
}

Follow this and additional works at: https://aah.org/jpcrr

Part of the Interprofessional Education Commons, and the Medical Humanities Commons

\section{Recommended Citation}

In gratitude of 2021 JPCRR peer reviewers. J Patient Cent Res Rev. 2022;9:83. doi: 10.17294/

2330-0698.1946

Published quarterly by Midwest-based health system Advocate Aurora Health and indexed in PubMed Central, the Journal of Patient-Centered Research and Reviews (JPCRR) is an open access, peer-reviewed medical journal focused on disseminating scholarly works devoted to improving patient-centered care practices, health outcomes, and the patient experience. 


\section{In Gratitude of 2021 JPCRR Peer Reviewers}

Journal of Patient-Centered Research and Reviews calls on a diverse stable of reviewers to evaluate and enhance original submissions to the journal.

Our editorial team gratefully acknowledges the following volunteer experts for their generous contributions during the past 12 months.

Amit Acharya

James Adefisoye

Monica Ajinkya

Andy Anderson

Deanna Attai

Sarang Baman

Dennis Baumgardner

Eric Beck

Michelle Braccioforte

Laura Brady

John Brill

Peter Bulova

Scott Cameron

Gene Carroccia

Jane Chen

Brian Chicoine

Ron Cisler

Michelle Corbett

Callie Cox Bauer

Michelle Crane

James Dolbow

Lee Dresang

Alison Essary

Adam Farmer

Mustafa Farooque

Veronica Fitzpatrick
Marie Forgie

Sheldon Garrison

Nicole Gill

Michael Gionfriddo

Nicole Glowacki

Sarah Greene

Robert Greenlee

Ted Gronski

Joe Grundle

Inga Gruß

David Hamel

Erin Harvey

Julian Hong

Loretta Hsueh

Anna Huppler

Gaurav Jain

Alonzo Jalan

Anne Jones

Christopher Kabir

Ariba Khan

Rasha Khatib

Jillene Kogan

Rebecca Koscik

Fabiana Kotovicz

Bradley Kruger

Marianna LaNoue
Eric Larson

Melissa Lemke

Morgan Lively

William Lobb

Emily Malloy

Barry Martin

William Michael

Alyssa Mohorek

Bruce Morgenstern

Christopher Morley

George Morris, III

Michael Mullane

Emily Murray

Nikhil Narang

Hemalatha Narayanasamy

Donald Nease, Jr.

Kari Nelson

Vani Nilakantan

Kushal Patel

Sunil Pauwaa

Vina Raj

Amit Ray

Jon Richards

Mark Robinson

Steve Rommelfanger

Ajay Sahajpal
James Sanders

Laura Schad

Sarina Schrager

Rebecca Schultz

Deborah Simpson

David Smith

Jessica Smith

Paul Smith

Victoria Smoter

Leif Solberg

Alexandrea Spindler

Mary Stephens

Lisa Sullivan Vedder

Muthana Sweis

Michael Thompson

Judy Tjoe

Nick Turkal

Daniela Uribe-Cano

Cynthia Valentin

Gabriela Vazquez-Benitez

Vida Vizgirda

Mindy Waite

John Westfall

Frank Zimmerman

Viviana Zlochiver

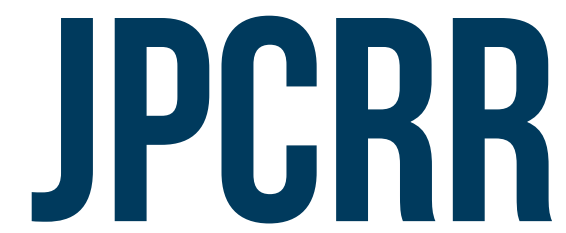

Journal of Patient-Centered Research and Reviews 